\title{
Photorefractive Effect of Liquid Crystalline Materials
}

\author{
Takeo SASAKI ${ }^{\dagger}$ \\ Department of Chemistry, Faculty of Science, Science University of Tokyo, \\ 1-3 Kagurazaka, Shinjuku-ku, Tokyo 162-8601, Japan
}

(Received July 4, 2005; Accepted July 9, 2005; Published November 15, 2005)

\begin{abstract}
This paper reviews our recent work on the photorefractive effect in liquid crystalline materials. The photorefractive effect is defined as the optical modulation of the refractive index of a medium as a result of various processes. The interference of two laser beams in a photorefractive material establishes a refractive index grating. This phenomenon enables the creation of different types of photonic applications. Recently, the development of organic photorefractive materials has been attracting great interest. Liquid crystalline materials are a strong candidate for practical photorefractive materials and application. In this paper, the photorefractivity of liquid crystalline polymers and ferroelectric liquid crystals is described. [DOI 10.1295/polymj.37.797]

KEY WORDS Photorefractive Effect / Liquid Crystalline Polymers / Ferroelectric Liquid Crystals / Two Beam Coupling / Gain Coefficient / Dynamic Holograms / Motion Mode Holograms /
\end{abstract}

The photorefractive effect is a phenomenon wherein a change in the refractive index of a material is induced by the absorption of light. The change in refractive index through the photorefractive effect occurs only within the interference fringes of incident laser beams. When laser beams interfere in a photorefractive material, charge separation occurs between the light and the dark positions of the interference fringes. A space-charge field, an internal electric field, develops at the areas between the light and the dark positions. The refractive index of the corresponding areas is changed through an electro-optic effect. Thus, a refractive index grating is formed at the interference fringes.

Dynamic volume-holograms are easily formed through the photorefractive effect, and this has direct applicability in photonics, including optical image processing, parallel optical logic, fringe recognition, and phase conjugation. The photorefractive effect was first observed in the inorganic crystal lithium niobate in $1967 .{ }^{1}$ It was also subsequently observed in an organic material in $1990 .^{2}$ Several reviews on the development of photorefractive materials have been published. ${ }^{3-7}$ The photorefractive effect has been reported in several organic materials since 1990, such as glassy polymers ${ }^{8-23}$ low-molecular-weight nematic liquid crystals, ${ }^{24-28}$ liquid crystalline polymers, ${ }^{29-35}$ ferroelectric liquid crystals, ${ }^{36-42}$ polymer/liquid crystal composites ${ }^{43-55}$ and amorphous compounds, ${ }^{56,57}$ and extremely high photorefractivity values have been achieved in organic polymeric materials. The high photorefractivity in polymeric materials arises from changes in the orientations of the chromophores induced by the internal electric field, termed orientational enhancement. ${ }^{4,15,17}$ The photorefractive efficiency of organic materials is several times that of inorganic photorefractive crystals. In addition, organic polymers can easily be processed into films and fibers. This is a significant advantage for applications in photonics.

\section{Mechanism of the Photorefractive Effect}

A photorefractive material exhibits both photoconductivity as well as an electro-optic effect. This means that a material which possesses both of these properties exhibits photorefractivity. An organic photorefractive material is composed of a $\mathrm{D}-\pi-\mathrm{A}$ chromophore, a photoconductive compound and an electron trap reagent. When two coherent laser beams interfere in a photorefractive material, a refractive index grating is formed. Figure 1 shows the interference fringes formed with light and dark. In the light areas, photoinduced charge generation occurs, and free electrons and holes are generated. While the electrons are trapped at the trap sites, the positive charges thermally diffuse to all parts of the material. As a result, the light positions of the fringes are negatively charged and the dark positions are positively charged. An electric field is thus induced between the light positions and the dark positions. This electric field is termed the "internal electric field". Because the material contains $\mathrm{D}-\pi-\mathrm{A}$ chromophores that possess large dipole moments, the orientations of the chromophores are altered by the presence of the internal electric field.

${ }^{\dagger}$ To whom correspondence should be addressed (Tel: +81-3-5228-8277, Fax: +81-3-3235-2214, E-mail: sasaki@ rs.kagu.tus.ac.jp). 
(a)

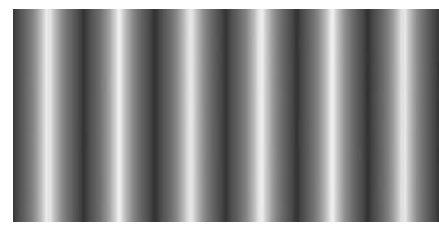

Interference

(b)

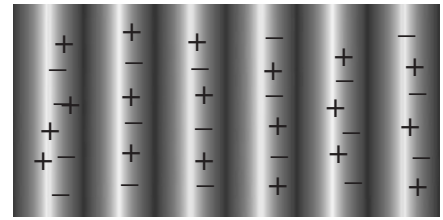

Charge generation

(c)

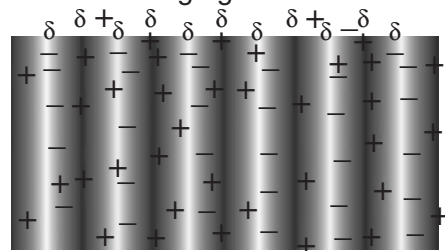

Charge transport,

d)
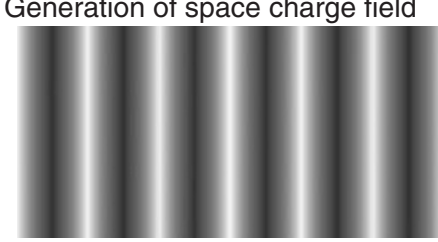

Electro-optic index modulation Formation of refractive index grating

Figure 1. Schematic illustration of the mechanism of the photorefractive effect: (a) Two laser beams interfere in the photorefractive material; (b) charge generation occurs at the light areas of the interference fringes; (c) while the electrons are trapped at the trap sites in the light areas, holes migrate by diffusion or drift in the presence of an external electric field and generate an internal-electric field between the light and dark positions; (d) the refractive index of the corresponding area is altered by the internal electric field.

The apparent refractive index of the areas between the light and the dark positions is changed through reorientation of the chromophores. Thus, a refractive index grating is formed.

It is characteristic of the photorefractive effect that the phase of the refractive index grating is $\pi / 2$-shifted from the interference fringes. When the material is photochromic, a photochemical reaction takes place at the light areas, and a refractive index grating with the same phase as that of the interference fringe is formed (Figure 2). However, if the material is photorefractive, the phase of the refractive index grating is shifted from that of the interference fringes, and this affects the propagation of the two beams. Beam 1 is energetically coupled with beam 2 for the two laser beams. Consequently, the apparent transmitted intensity of beam 1 increases and that of beam 2 decreases, as shown in Figure 3. This phenomenon is termed asymmetric energy exchange in the two-beam cou- (a) Photochromic

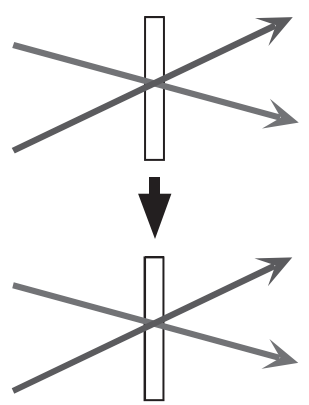

Interference fringe
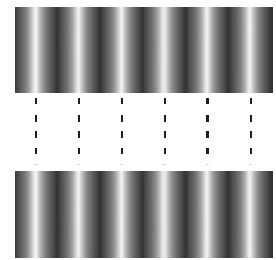

Refractive index grating

(b) Photorefractive

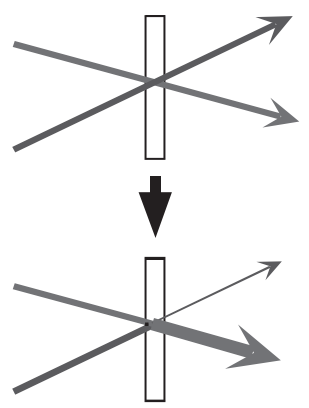

Interference fringe
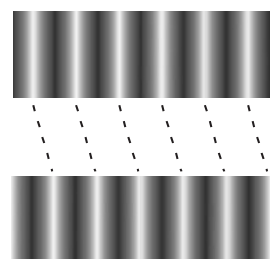

Refractive index grating

Asymmetric Energy Exchange

Figure 2. (a) Photochromic grating and (b) Photorefractive grating.
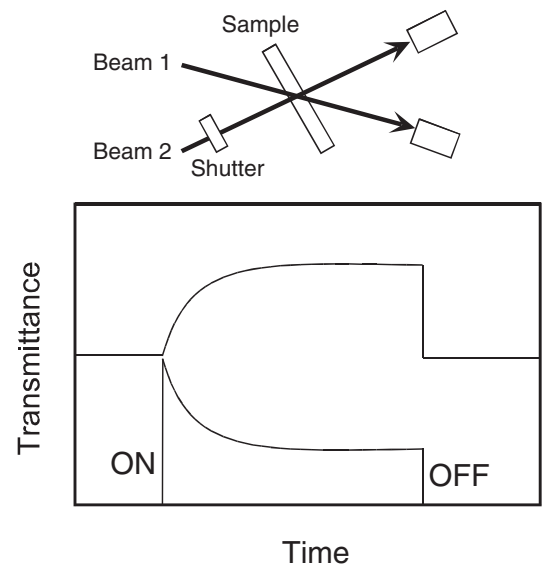

Figure 3. Two-beam coupling signal.

pling experiment. The photorefractivity of a material is confirmed by the occurrence of this asymmetric energy exchange.

\section{Measurement of Photorefractivity}

The photorefractive effect is evaluated by a twobeam coupling method and also by a four-wave mixing experiment. ${ }^{3-7}$ Figure $4 \mathrm{a}$ shows a schematic illustration of the experimental setup for the two-beam coupling method. A p-polarized beam from a laser is divided into two beams by a beam splitter, and the beams are interfered within the sample film. An electric field is applied to the sample using a high voltage 
(a)

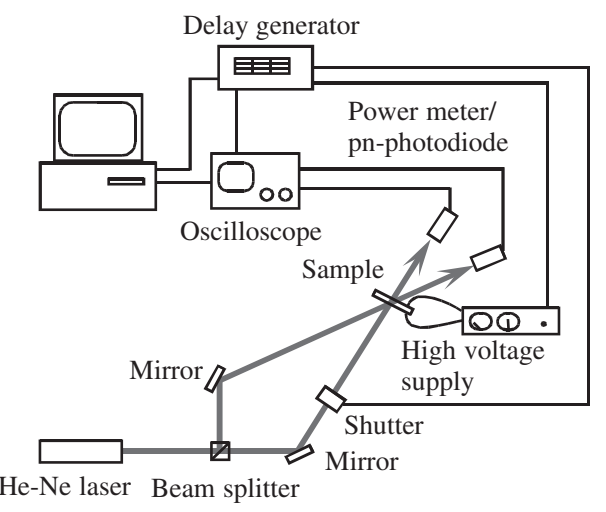

(b)

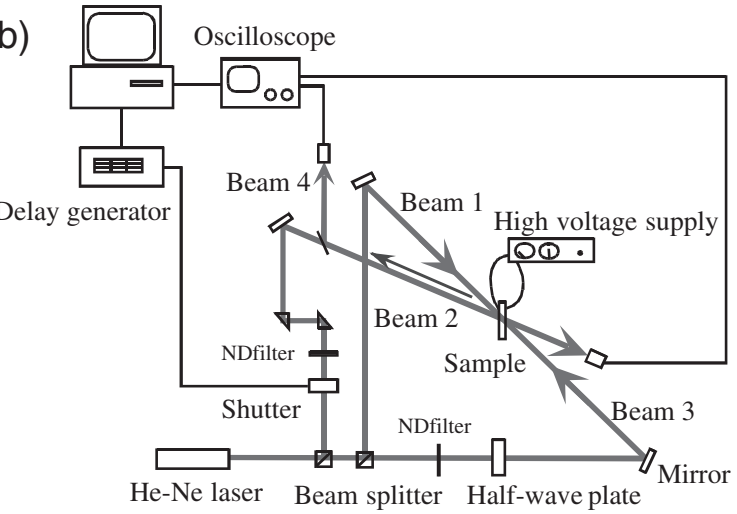

Figure 4. Schematic illustrations of the experimental set-up for (a) two-beam coupling and (b) four-wave-mixing techniques.

supply unit. This external electric field is applied in order to increase the efficiency of charge generation. The change in the transmitted beam intensity is monitored. If a material is photorefractive, an asymmetric energy exchange is observed.

The magnitude of photorefractivity is evaluated using a parameter called the gain coefficient, which is calculated from the change in transmitted intensity of the laser beams induced through the two-beam coupling. In order to calculate the two-beam coupling gain coefficient, it must be determined whether the diffraction condition is in the Bragg regime or in the Raman-Nath regime. These diffraction conditions are distinguished by a dimensionless parameter $\mathrm{Q}{ }^{4}$

$$
\mathrm{Q}=2 \pi \lambda L / n \Lambda^{2}
$$

Q $>1$ is defined as the Bragg regime of optical diffraction. In this regime, multiple scattering is not permitted, and only one order of diffraction is produced. Conversely, $\mathrm{Q}<1$ is defined as the Raman-Nath regime of optical diffraction. In this regime, many orders of diffraction can be observed. Usually, $Q>10$ is required to guarantee that the diffraction is entirely in the Bragg regime. When the diffraction is in the Bragg diffraction regime, the two-beam coupling gain coefficient $\Gamma\left(\mathrm{cm}^{-1}\right)$ is calculated according to the following equation:

$$
\Gamma=\frac{1}{D} \ln \left(\frac{g m}{1+m-g}\right)
$$

where $D=L / \cos (\theta)$ is the interaction path for the signal beam $(L=$ sample thickness, $\theta=$ propagation angle of the signal beam in the sample), $g$ is the ratio of the intensities of the signal beam behind the sample with and without a pump beam, and $m$ is the ratio of the beam intensities (pump/signal) in front of the sample.

A schematic illustration of the experimental setup for the four-wave mixing experiment is shown in Figure 4b. S-polarized writing beams are interfered in the sample film and the diffraction of a p-polarized probe beam, counter-propagating to one of the writing beams, is measured. The diffracted beam intensity is typically measured as a function of time, applied (external) electric field, writing beam intensities, etc. The diffraction efficiency is defined as the ratio of the intensity of the diffracted beam and the intensity of the probe beam that is transmitted when no grating is present in the sample due to the writing beams. In probing the grating, it is important that beam 3 does not affect the grating or interact with the writing beams. This can be ensured by making the probe beam much weaker than the writing beams, and by having the probe beam polarized orthogonal to the writing beams.

Photorefractive Effect in Glassy Polymeric Materials

The photorefractive effect in an organic polymer, shown in Figure 5, was first reported in 1992. ${ }^{8}$ A film of polyvinylcarbazole (PVK) doped with DEANST
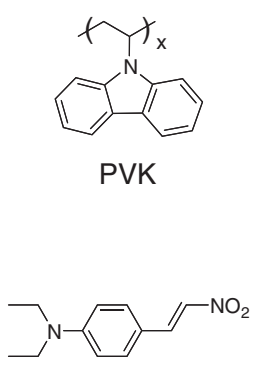

DEANST
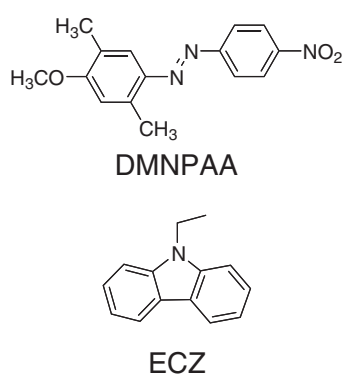

Figure 5. Examples of compounds used in photorefractive polymers. 

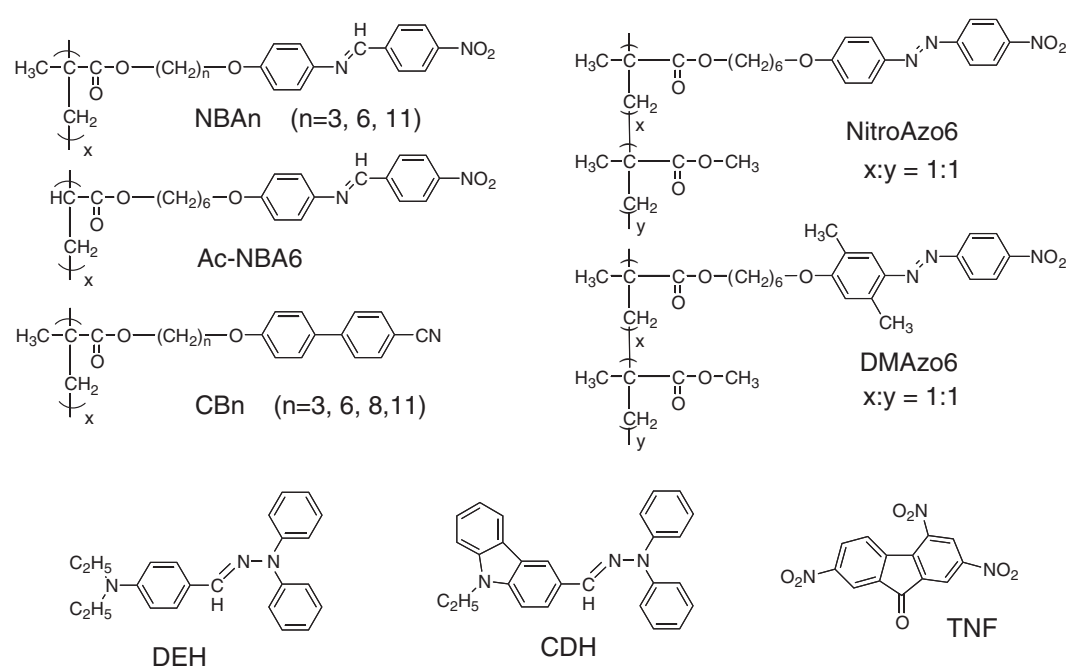

Figure 6. Structures of the LC polymers.

and $\mathrm{C}_{60}$ exhibited a diffraction efficiency of $\sim 10^{-5} \%$. DEANST was designed to exhibit a high Pockels constant. A number of studies on the photorefractivity in polymers were conducted after this initial report, and a diffraction efficiency of $\sim 6 \%$ was achieved in 1993..$^{9}$ In 1994, a very sensitive paper was reported by Meerholz et al. in Nature. ${ }^{12}$ A mixture of PVK, DMNPAA, TNF and ECZ (a plasticizer) exhibited a diffraction efficiency of nearly $100 \%$ in a $100 \mu \mathrm{m}$ sample. The electro-optic properties (such as Pockels constant) of the dopant chromophores could not account for such a large diffraction efficiency. A possible explanation was that the high diffraction efficiency could be attributed to an orientational birefringence contribution caused by an internal electric field.

The 1994 paper by Meerholz et al. was a turning point in the development of photorefractive polymers since it completely changed the way chromophores began to be designed. The Pockels effect was no longer the main driver, rather, the orientational birefringence became important. Studies on low- $T_{\mathrm{g}}$ materials began to attract great interest. Plasticizers are commonly added to a photorefractive composite to lower the $T_{\mathrm{g}}$. The concentration of the plasticizer reaches a few tens $\%$. In order to retain high photoconductivity, ECZ is widely used. For using ECZ as a plasticizer, even though larger amounts were needed to lower the $T_{\mathrm{g}}$ to the desired value, the charge transport properties did not deteriorate. The development of photorefractive glassy polymers have been comprehensively reviewed by Moerner et al. ${ }^{5,7}$

\section{PHOTOREFRACTIVITY IN LIQUID CRYSTALLINE POLYMERS}

\section{Photorefractivity in Isotropic Phase in LC Polymers}

The photorefractive effect in LC polymers was first

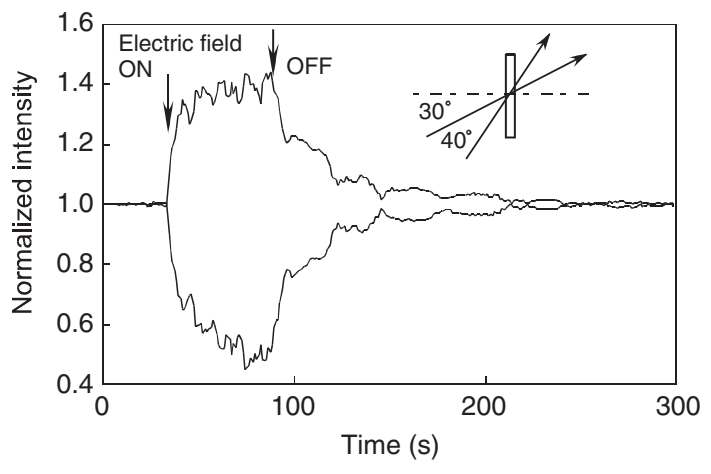

Figure 7. Typical example of asymmetric energy exchange observed in a two-beam coupling experiment for NBA6 doped with $30 \mathrm{wt} \% \mathrm{DEH}$ and $1 \mathrm{wt} \% \mathrm{TNF}$. An electric field of $30 \mathrm{~V} /$ $\mu \mathrm{m}$ was applied to the sample at $t=35 \mathrm{~s}$ and the field was cut off at $t=90 \mathrm{~s}$.

reported by the present Authors in 1999. ${ }^{30}$ The photorefractivity of a LC polymer, NBA6, shown in Figure 6, doped with $30 \mathrm{wt} \%$ DEH and $1 \mathrm{wt} \% \mathrm{TNF}$ was investigated. The photoconductive dopant, DEH, is not a liquid crystal. Therefore, the addition of 30 wt \% DEH disturbed the liquid crystal phase of the host polymer. The sample film was isotropic and transparent, and was sandwiched between two ITOglass plates with a film thickness of $100 \mu \mathrm{m}$. A typical example of the asymmetric energy exchange in a twobeam coupling experiment for this material is shown in Figure 7. The transmitted intensities of the laser beams through the sample film are shown as a function of time and on the application of a $30 \mathrm{~V} / \mu \mathrm{m}$ electric field to the sample. When the electric field was applied, the transmitted intensity of one beam increased while that of the other beam decreased. A diffraction efficiency of about $40 \%$ was observed in this case. When the field was turned off, the intensities of the two beams gradually returned to their original values 


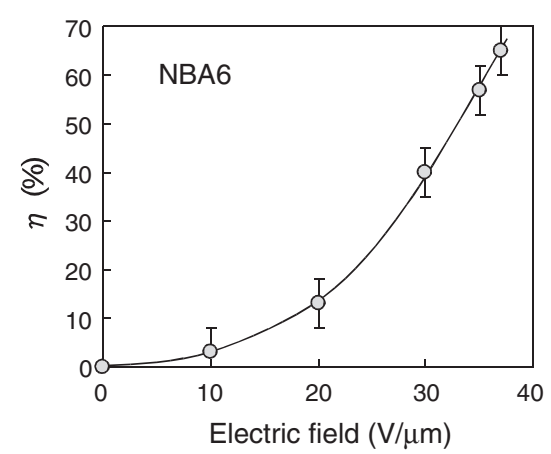

Figure 8. Dependence of diffraction efficiency on the applied electric field for NBA6 doped with $30 \mathrm{wt} \%$ DEH and $1 \mathrm{wt} \%$ TNF.

because of the relaxation of the $\mathrm{D}-\pi-\mathrm{A}$ chromophores.

The electric field dependence of the diffraction efficiency is shown in Figure 8. The diffraction efficiency increased with increasing strength of the applied electric field as a result of an increased amount of the generated charge. As shown in Figure 7, the diffracted beams appeared only in the presence of an applied electric field, eliminating the possibility of beam coupling due to thermal gratings. The diffraction efficiency of NBA6 was measured to be $65 \%$ at an applied voltage of $37 \mathrm{~V} / \mu \mathrm{m}$.

\section{Effect of Liquid Crystallinity}

The photorefractivity values of liquid crystalline polymers were compared with those of amorphous polymers. ${ }^{30-34}$ Ac-NBA6 (Figure 6) did not exhibit a liquid crystal phase even though its molecular structure is almost the same as that of NBA6. ${ }^{30}$ The only difference is the structure of the main chain. NBA6 is a methacrylate polymer and Ac-NBA6 is an acrylate polymer. The diffraction efficiencies of Ac-NBA6, methacrylate NBA6 and NBA3 are plotted as a function of the applied electric field in Figure 9. The twobeam coupling experiments were conducted under the condition of a transparent isotropic phase of the polymers at room temperature. The concentrations of DEH and TNF were 30 and $1 \mathrm{wt} \%$ respectively. The diffraction efficiency of Ac-NBA6 was found to be much smaller than that of NBA6, although the molecular weight was almost the same for these polymers and the glass transition temperature of Ac-NBA6 is lower than that of NBA6. The diffraction efficiency of the amorphous NBA3 polymer was also much smaller than that of NBA6. Thus, the photorefractivity is larger in the isotropic phase of the liquid crystalline polymer compared to that of the amorphous polymers.

The photorefractive properties of cyanobiphenyl PLCs were also investigated. ${ }^{30} \mathrm{CB} 6$ and CB8 exhibit both nematic and smectic phases. CB3 on the other hand exhibits only a nematic phase; however, the

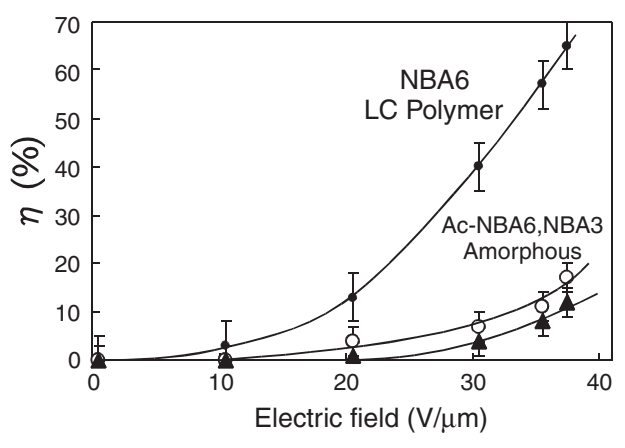

Figure 9. Dependence of diffraction efficiency on the applied electric field for NBAn doped with $30 \mathrm{wt} \% \mathrm{DEH}$ and $1 \mathrm{wt} \% \mathrm{TNF}$.

-, NBA6; O, Ac-NBA6, $\boldsymbol{\Delta}, \mathrm{NBA} 3$.

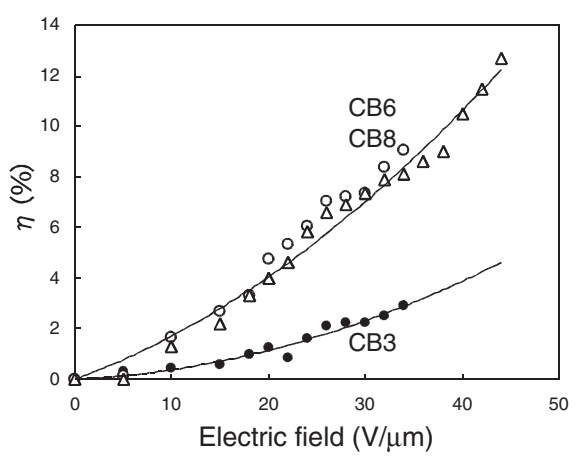

Figure 10. Dependence of diffraction efficiency on the applied electric field for CBn doped with $30 \mathrm{wt} \% \mathrm{DEH}$ and $1 \mathrm{wt} \% \mathrm{TNF}$. O, CB6; $\triangle$, CB8, •, CB3.

order parameter is very small. CB3 is clearly inferior to CB6 and CB8 in terms of the ability to form a liquid crystal phase. The electric field dependence of the diffraction efficiencies of CB6, CB8 and CB3, is shown in Figure 10. The concentrations of DEH and TNF were 30 and $1 \mathrm{wt} \%$ respectively. At this DEH concentration, these polymers did not exhibit a liquid crystal phase. The diffraction efficiency of $\mathrm{CB} 3$ was several times smaller than that of CB6 and CB8, although the CB3 sample was transparent and the glass transition temperature of $\mathrm{CB} 3$ was almost the same as that of CB6.

A 1:1 copolymer of methylmethacrylate and 4methacryloyloxyhexyloxy-4'-nitroazobenzene (NitroAzo6) exhibits a nematic liquid crystal phase, whereas a 1:1 copolymer of methylmethacrylate and 4'-methacryloyloxyhexyloxy-2'5'-dimethyl-4-nitroazobenzene (DMAzo6) does not exhibit a liquid crystal phase. The diffraction efficiencies of the polymers NitroAzo6 and DMAzo6 mixed with photoconductive compounds (CDH and TNF) were investigated. ${ }^{34}$ The diffraction efficiency of DMAzo6 was found to be much smaller than that of NitroAzo6, although their molecular weights are almost the same and DMAzo6 exhibits a lower glass transition temperature than NitroAzo6. It can be concluded that the photorefractivity becomes 
(a)

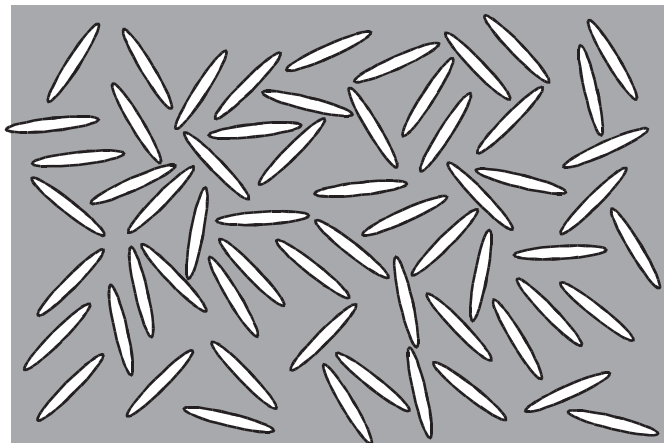

(b)

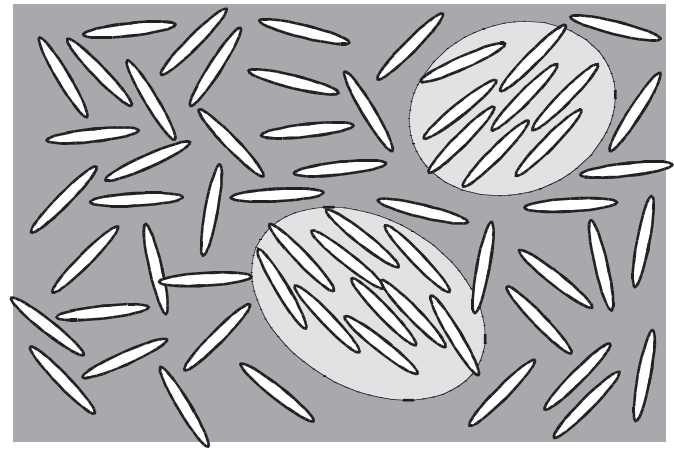

Figure 11. Illustrations of (a) amorphous polymers and (b) isotropic phase of LC polymers.

large in the isotropic phase of a liquid crystalline polymer in comparison with polymers with low liquid crystallinity. These results indicate that the isotropic phase of liquid crystalline polymers is not the same as that of amorphous polymers.

As the molecular structure of NBA6 is almost identical to that of Ac-NBA6, the enhanced photorefractivity in the former likely arises from the morphology of the isotropic phase. The isotropic phase of a LC is believed to include orientations of mesogens that are completely random. However, in the case of the isotropic phase of the NBA6/DEH mixture, the orientations of mesogens are randomized not by the rising temperature, but by a lowering of the phase transition temperature through the introduction of an impurity (DEH) into the LC polymer. The formation of an ordered structure is thermodynamically prevented by the presence of the impurity. However, local concentration of DEH is not unity throughout a highly viscous medium such as the polymer film. Therefore, some fractions of the mesogens will have a tendency to form locally ordered structures in the microscopic region, as shown in Figure 11. It is likely that the electro-optic effect was enhanced by the presence of such a microdomain structure thereby leading to larger photorefractivity.

\section{Photorefractive Effect in LC Polymers Containing Hydrogen-Bonding Moiety}

The enhancement of photorefractivity in LC poly-

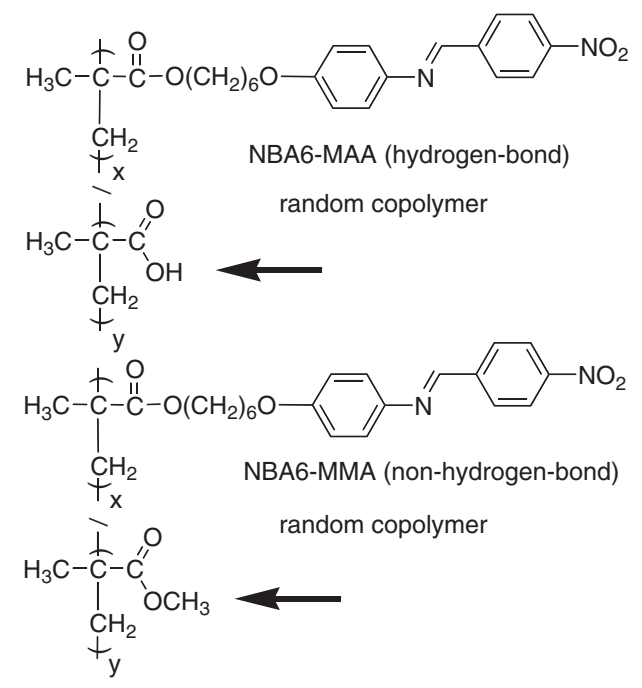

Figure 12. Molecular structures of NBA6-MAA and NBA6MMA.

mers is considered to arise from the microscopicallyordered structure of component chromophores in the isotropic phase. If the microscopically-ordered structure plays an important role in the enhancement of the photorefractive effect, the introduction of a hydrogen-bonding moiety into the polymer would significantly affect the photorefractivity. To investigate this possibility, the photorefractivity of $\mathrm{D}-\pi-\mathrm{A}$ polymers possessing a hydrogen-bond-formable moiety was compared with that of polymers without hydrogenbonding moieties. ${ }^{35}$ The photorefractive properties of copolymers of a nitrobenazylideneaniline monomer and methacrylic acid (NBA6-MAA, see Figure 12) were investigated. The carboxylic acid moieties are capable of forming hydrogen-bonds and this introduces micro-domains in the polymer film. Copolymers of nitrobenzylidenaniline monomer and methyl methacrylate (NBA6-MMA) were used as reference polymers.

The glass transition temperature and nematic-isotropic phase transition temperature are both higher in NBA6-MAA when compared to NBA6-MMA. Conformational changes of the main chain of NBA6MAA are restricted by the hydrogen-bonding and could lead to higher phase transition temperatures. The temperature dependence of the diffraction efficiencies of NBA6-MAA and NBA6-MMA mixed with $30 \mathrm{wt} \% \mathrm{CDH}$ and $1 \mathrm{wt} \% \mathrm{TNF}$ are shown in Figure 13. The diffraction efficiency of the non-hydrogen-bonding polymer NBA6-MMA increased with increasing temperature around $T_{\mathrm{g}}$ and decreased at higher temperatures. However, the hydrogen-bonding polymer NBA6-MAA exhibited larger diffraction efficiencies at temperatures below $T_{\mathrm{g}}$ which decreased as the temperature was raised above $T_{\mathrm{g}}$. For the copolymerization ratios considered, the diffraction efficien- 

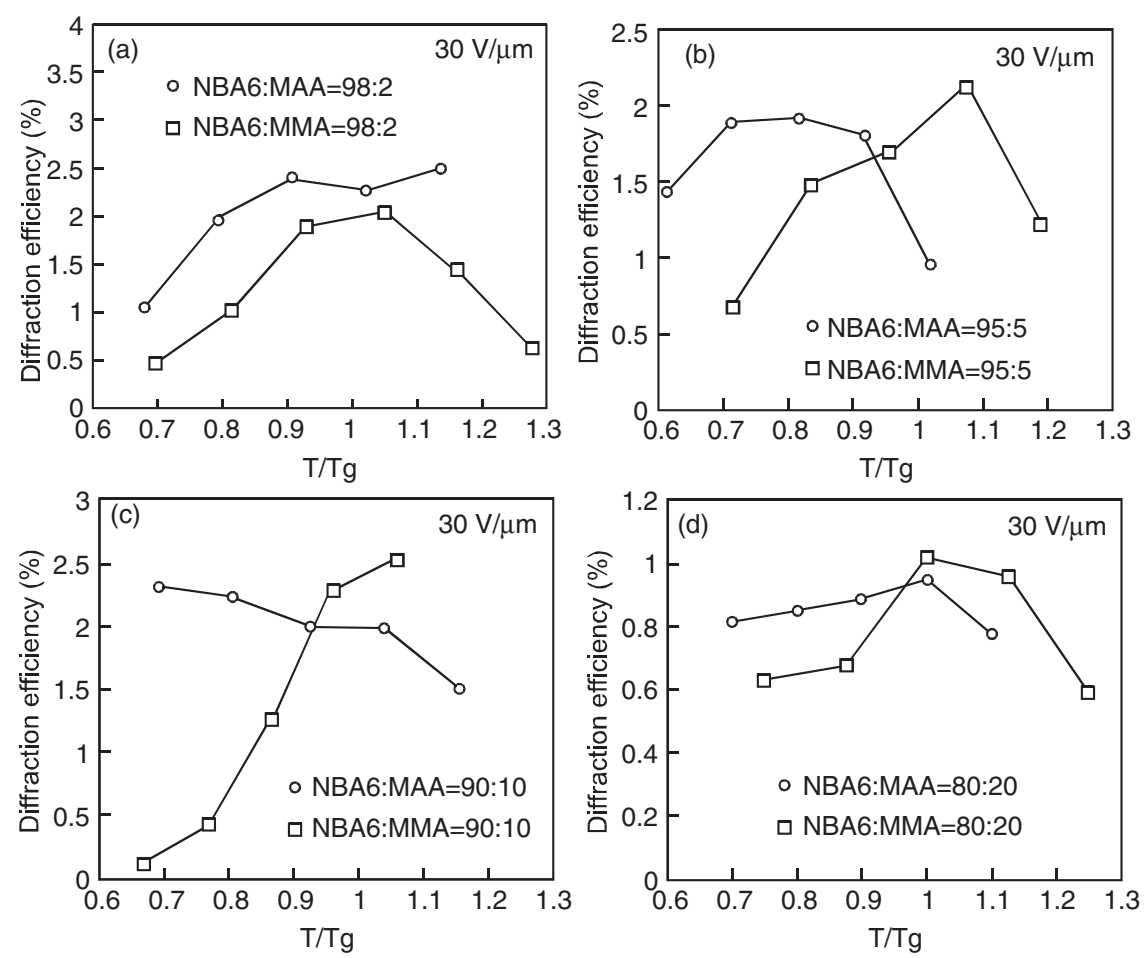

Figure 13. Temperature dependence of the diffraction efficiencies of NBA6/CDH/TNF mixture measured for different strengths of the external electric field. (a) copolymerization ratio $=98: 2$; (b) copolymerization ratio $=95: 5$; (c) copolymerization ratio $=90: 10$; $(d)$ copolymerization ratio $=80: 20$.

cies of NBA6-MAA at temperatures $T / T_{\mathrm{g}}=0.7-0.9$ were much larger than those of NBA6-MMA. The larger diffraction efficiencies in NBA6-MAA are considered to originate from the large mobility of the side-chain $\mathrm{D}-\pi-\mathrm{A}$ chromophores below $T_{\mathrm{g}}$, as well as due to an enhancement in the electro-optic effect as a result of the presence of micro-domains. A change in the refractive index for the photorefractive effect in organic polymers arises primarily from the orientational change of $\mathrm{D}-\pi-\mathrm{A}$ chromophores. However, in common photorefractive polymers, the mobility of side-chain $\mathrm{D}-\pi-\mathrm{A}$ chromophores in the film is low at temperatures below $T_{\mathrm{g}}$. It was likely that the mobility of $\mathrm{D}-\pi-\mathrm{A}$ chromophores in NBA6-MAA films at temperatures below $T_{\mathrm{g}}$ was enhanced by the presence of the hydrogen-bonding moiety.

The conformation of the NBA6-MAA main chain is restricted by the hydrogen-bonding and is likely to be in a stressed state. This results in a larger inner free volume around the chromophores, as shown in Figure 14. The glass transition temperature, below which the main chains freeze, was higher in NBA6MAA. However, the free volumes around the sidechains likely swelled because of the stressed conformation of the main chains. When the temperature was increased above $T_{\mathrm{g}}$, the hydrogen-bonding was broken and the conformation of the main chain relaxed to the thermally stable state. The side-chains are closely packed and this leads to smaller free vol- umes around the $\mathrm{D}-\pi-\mathrm{A}$ chromophores. Moreover, the microdomains formed via the hydrogen-bonding are disorganized at temperatures above $T_{\mathrm{g}}$ and this lowers the photorefractivity.

\section{Memory Effect in Photorefractive LC Polymers}

The memory effect of photorefractivity was investigated in a LC polymer. ${ }^{33}$ Two laser beams were interfered in a mixture of $\mathrm{CBn}, \mathrm{CDH}$ and TNF, and the diffraction of a probe beam was measured using a fourwave-mixing configuration. When irradiation of the sample by the writing beams was stopped, the intensity of the diffraction signal dropped sharply, but did not drop to zero immediately. A weak signal could still be observed after both writing beams were cut off, and this signal was larger for lower $\mathrm{CDH}$ concentrations. This persistent diffraction signal was observed in polymers with spacer chains longer than $\mathrm{n}=11$. Figure 15 shows the diffraction signals observed in the CB6 and CB12 samples. This memory effect of the diffraction in LC polymer composites arises from the persistence of the orientational grating and its origin has not been identified. However, it is believed that the orientational grating of the mesogens may be stabilized by the presence of the polymer, more specifically for $\mathrm{CBn}$, by the interaction between the chromophores and the alkyl chain of the polymer. The persistence of the diffraction in $\mathrm{CBn}$ is not observed when the temperature is increased above 

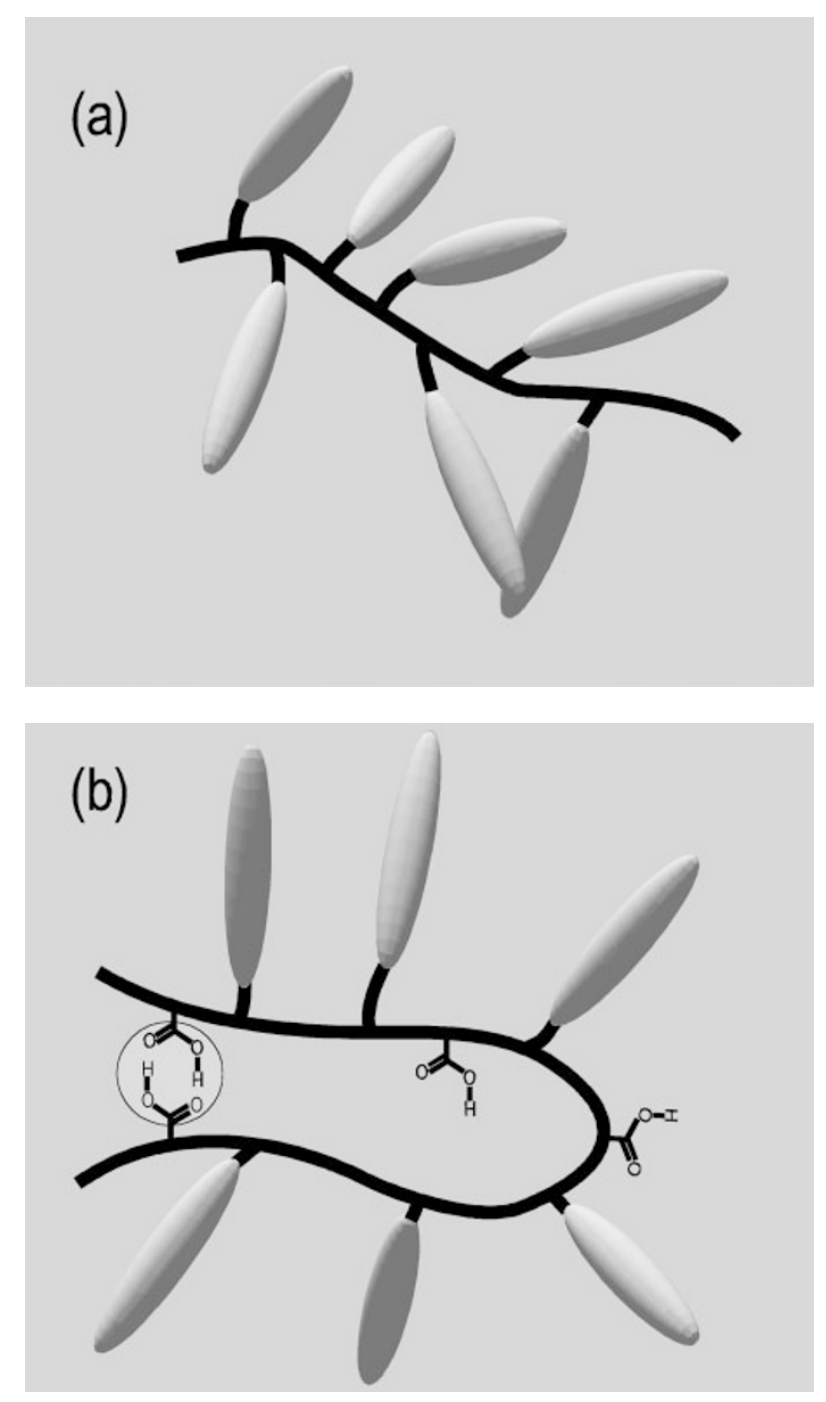

Figure 14. Illustration of (a) a polymer without hydrogenbonding, and (b) a polymer with a hydrogen-bonding moiety.

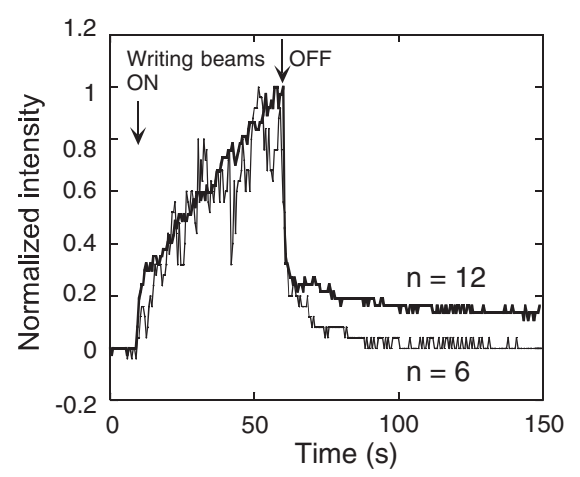

Figure 15. Diffraction signals of $\mathrm{CB} 12$ and $\mathrm{CB} 6$ mixed with $40 \% \mathrm{CDH}$ and $1 \mathrm{wt} \% \mathrm{TNF}$.

$45^{\circ} \mathrm{C}$. It is believed that the orientational grating is disturbed by the thermal motion of the cyanobiphenyl chromophores. At higher $\mathrm{CDH}$ concentrations, $T_{\mathrm{g}}$ decreases as the orientational grating cannot be sustained because of thermal motion of the mesogens.
In addition, the interaction between the chromophores and the alkyl chains are reduced by the presence of the dopant. Thus, the persistence of the orientational grating is enhanced in polymers with longer spacer chains and lower $\mathrm{CDH}$ concentrations.

\section{PHOTOREFRACTIVITY IN FERROELECTRIC LIQUID CRYSTALS}

\section{Photorefractive Effect of Ferroelectric LCs}

The photorefractive effect in low-molecular-weight nematic liquid crystals has been investigated, ${ }^{24-28}$ and photo-induced changes in refractive index in such crystals has been shown to be caused by changes in the orientation of the liquid crystal molecules induced by the internal electric field. The effect of reorientation of liquid crystal molecules on the apparent refractive index is particularly strong. Despite the apparent advantages of organic photorefractive materials, their response times are too slow (usually $\sim 100 \mathrm{~ms}$ ) for practical applications, which can be attributed to the slow reorientation of chromophores. The use of high- $T_{\mathrm{g}}$ polymers has been investigated as one means of addressing this problem. ${ }^{20,21}$ In such stiff materials, the reorientation of chromophores is restricted, and refractive index modulation is solely due to the Pockels effect. Although rapid response times have been achieved in these materials, the reported diffraction efficiencies are lower than in low- $T_{\mathrm{g}}$ materials due to the $a b-$ sence of a supplementary orientational mechanism.

In order to obtain faster photorefractive responses, ferroelectric liquid crystals (FLCs) were investigated. $^{36-42}$ FLCs are classified as chiral smectic C $\left(\mathrm{SmC}^{*}\right)$ liquid crystals that form a layered structure. ${ }^{58-60}$ In the $\mathrm{SmC}^{*}$ phase, the long axis of each FLC molecule tilts from the layer normal. Spontaneous polarization (bulk polarization) occurs when the FLCs with the $\mathrm{SmC}^{*}$ phase are sandwiched between glass plates within a thickness range of $2-10 \mu \mathrm{m}$, in a surface stabilized state. When a DC electric field is applied to an FLC, the direction of this spontaneous polarization is governed by the polarity of the applied DC field. The tilting direction of the molecular long axis of FLC also switches in accordance with the direction of the spontaneous polarization. Since the switching of FLC molecules is due to the response of bulk polarization, the switching is extremely fast. Figure 16 shows a schematic illustration of the mechanism of the photorefractive effect in FLCs. In this scheme, the internal electric field alters the direction of spontaneous polarization in the areas between the light and dark positions of the interference fringes, which induces a periodic change in the orientation of the FLC molecules. This is different from the processes that occur in other photorefractive materials 
a)

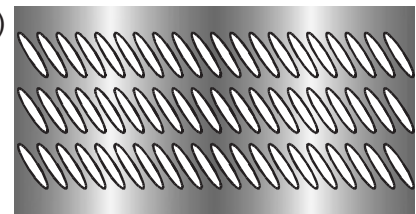

Interference in FLC

b)

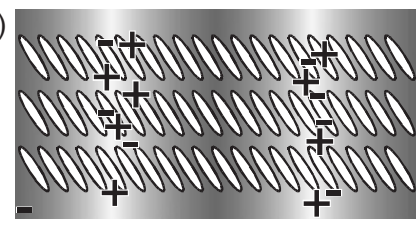

Charge generation

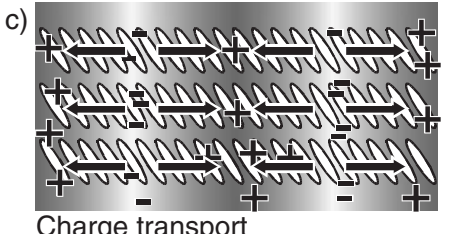

Charge transport

Generation of internal electric field

d)

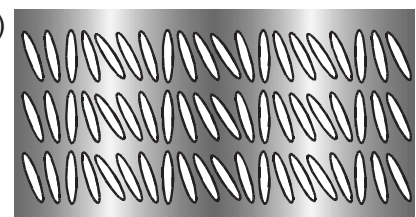

Change in orientation of

FLC molecules

Figure 16. Schematic illustration of the mechanism of the photorefractive effect in FLCs. (a) Two laser beams interfere in the surface-stabilized state of the FLC/photoconductive compound mixture; (b) charge generation occurs at the bright areas of the interference fringes; (c) while electrons are trapped at the trap sites in the bright areas, holes migrate by diffusion or drift in the presence of an external electric field to generate an internal electric field between the bright and dark positions; (d) the orientation of the spontaneous polarization vector (i.e., orientation of mesogens) is altered by the internal electric field.

wherein the molecular dipoles, rather than the bulk polarization, respond to the internal electric field.

\section{Two-beam Coupling Experiment Using FLCs}

The photorefractive effect of a mixture of FLC/ $\mathrm{CDH} / \mathrm{TNF}$ was measured in a two-beam coupling experiment using a $488 \mathrm{~nm} \mathrm{Ar}^{+}$laser. $^{36-41}$ A commercially available FLC, SCE8, was used. The concentrations of $\mathrm{CDH}$ and TNF were 2 and $0.1 \mathrm{wt} \%$ respectively. The samples were injected into a $10-\mu \mathrm{m}$-gap glass cell equipped with $1 \mathrm{~cm}^{2}$ ITO electrodes and a polyimide alignment layer. An electric field of 0-10 $\mathrm{V} / \mu \mathrm{m}$ was applied to the sample. Figure 17 shows a typical example of the asymmetric energy exchange observed in the SCE8/CDH/TNF sample under an applied DC electric field of $0.1 \mathrm{~V} / \mu \mathrm{m}$. Interference of the divided beams in the sample resulted in increas-

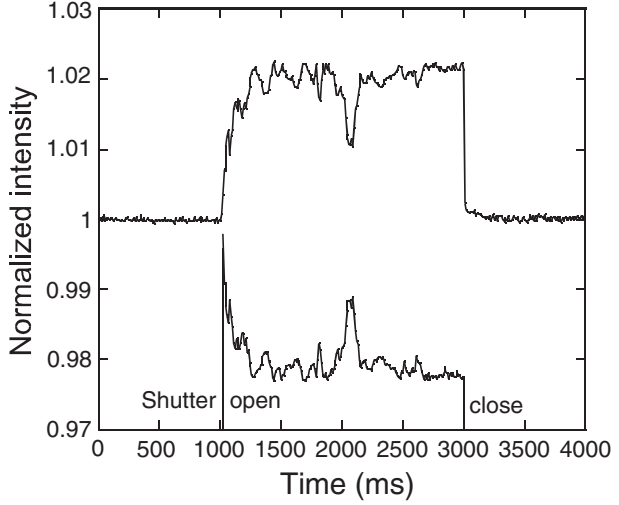

Figure 17. Typical example of asymmetric energy exchange observed in SCE8 mixed with $2 \mathrm{wt} \% \mathrm{CDH}$ and $0.1 \mathrm{wt} \%$ TNF. An electric field of $+0.3 \mathrm{~V} / \mu \mathrm{m}$ was applied to the sample.
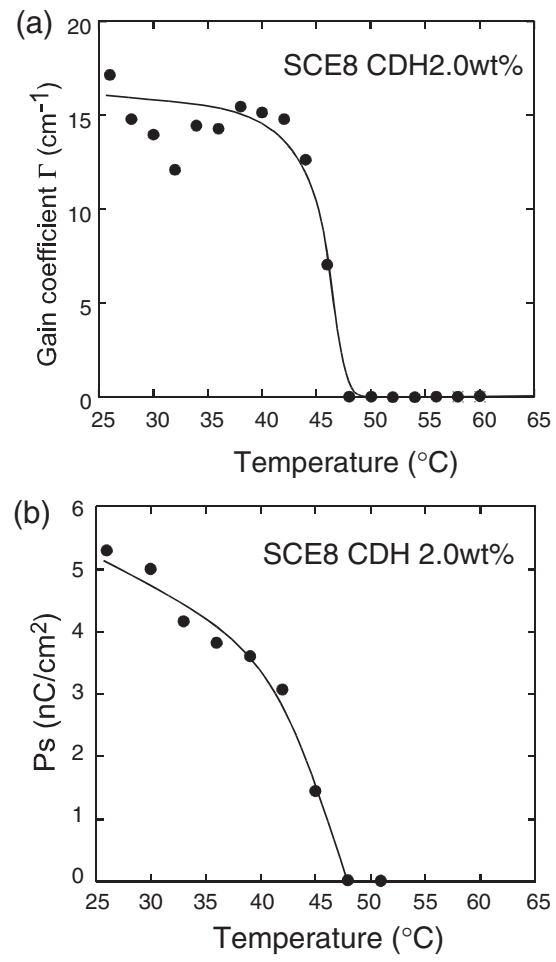

Figure 18. Temperature dependence of (a) gain coefficient and (b) spontaneous polarization of SCE8 mixed with $2 \mathrm{wt} \%$ $\mathrm{CDH}$ and $0.1 \mathrm{wt} \%$ TNF. For two-beam coupling experiments, an electric field of $0.1 \mathrm{~V} / \mu \mathrm{m}$ was applied to the sample.

ed transmittance of one beam and decreased transmittance of the other. The change in transmitted intensities of the two beams is completely symmetric, as can be seen in Figure 17. This indicates that the phase of the refractive index grating is shifted from that of the interference fringes. ${ }^{1-3}$ The grating formation was within the Bragg diffraction regime, ${ }^{2}$ and no higher order diffraction was observed under the conditions used.

The temperature dependence of the gain coefficient of SCE8 doped with $2 \mathrm{wt} \% \mathrm{CDH}$ and $0.1 \mathrm{wt} \% \mathrm{TNF}$ is shown in Figure 18a. Asymmetric energy exchange 
Table I. Physical properties of the FLCs investigated

\begin{tabular}{lccccc}
\hline FLC & $\begin{array}{c}\text { Ps at } \\
25^{\circ} \mathrm{C} \\
\left(\mathrm{nC} / \mathrm{cm}^{2}\right)\end{array}$ & $\begin{array}{c}\text { Phase transition temperature } \\
\left({ }^{\circ} \mathrm{C}\right)\end{array}$ & $\begin{array}{c}\text { Response } \\
\text { time } \tau^{\mathrm{b}} \\
(\mu \mathrm{s})\end{array}$ & $\begin{array}{c}\text { Rotational } \\
\text { viscosity } \gamma_{\phi} \\
(\mathrm{mPas})\end{array}$ & $\begin{array}{c}\text { Tilt angle } \\
(\text { deg. })\end{array}$ \\
\hline $015 / 000$ & 9 & $-\mathrm{Sc}^{*} 71 \mathrm{~S}_{\mathrm{A}} 83 \mathrm{~N}^{*} 86 \mathrm{I}$ & 70 & 60 & 24 \\
$015 / 100$ & 33 & $-\mathrm{Sc}^{*} 72 \mathrm{~S}_{\mathrm{A}} 83 \mathrm{~N}^{*} 86 \mathrm{I}$ & 21 & 80 & 23 \\
$016 / 000$ & -4.3 & $-\mathrm{Sc}^{*} 72 \mathrm{~S}_{\mathrm{A}} 85 \mathrm{~N}^{*} 93 \mathrm{I}$ & 70 & 61 & 25 \\
$016 / 030$ & -5.9 & $-\mathrm{Sc}^{*} 72 \mathrm{~S}_{\mathrm{A}} 85 \mathrm{~N}^{*} 93 \mathrm{I}$ & 47 & 82 & 25 \\
$016 / 100$ & -10.5 & $-\mathrm{Sc}^{*} 72 \mathrm{~S}_{\mathrm{A}} 85 \mathrm{~N}^{*} 94 \mathrm{I}$ & 20 & 60 & 27 \\
$017 / 000$ & 9.5 & $-\mathrm{Sc}^{*} 70 \mathrm{~S}_{\mathrm{A}} 76 \mathrm{~N}^{*} 87 \mathrm{I}$ & 93 & 47 & 26 \\
$017 / 100$ & 47 & $-\mathrm{Sc}^{*} 73 \mathrm{~S}_{\mathrm{A}} 77 \mathrm{~N}^{*} 87 \mathrm{I}$ & 23 & 116 & 27 \\
$018 / 000$ & 23 & $-\mathrm{Sc}^{*} 65 \mathrm{~S}_{\mathrm{A}} 82 \mathrm{~N}^{*} 88 \mathrm{I}$ & 59 & 68 & 22 \\
$018 / 100$ & 40 & $-\mathrm{Sc}^{*} 67 \mathrm{~S}_{\mathrm{A}} 82 \mathrm{~N}^{*} 89 \mathrm{I}$ & 30 & 97 & 23 \\
$019 / 000$ & 8.3 & $-\mathrm{Sc}^{*} 60 \mathrm{~S}_{\mathrm{A}} 76 \mathrm{~N}^{*} 82 \mathrm{I}$ & 262 & 37 & 19 \\
$019 / 100$ & 39 & $-\mathrm{Sc}^{*} 64 \mathrm{~S}_{\mathrm{A}} 78 \mathrm{~N}^{*} 87 \mathrm{I}$ & 53 & 75 & 20 \\
$\mathrm{SCE} 8$ & -4.5 & $-\mathrm{Sc}^{*} 60 \mathrm{~S}_{\mathrm{A}} 80 \mathrm{~N}^{*} 104 \mathrm{I}$ & 50 & 76 & 20 \\
M4851/000 & -4.0 & $-\mathrm{Sc}^{*} 64 \mathrm{~S}_{\mathrm{A}} 69 \mathrm{~N}^{*} 73 \mathrm{I}$ & 40 & - & 25 \\
M4851/050 & -14 & $-\mathrm{Sc}^{*} 65 \mathrm{~S}_{\mathrm{A}} 70 \mathrm{~N}^{*} 74 \mathrm{I}$ & 22 & 65 & 28 \\
\hline
\end{tabular}

${ }^{\mathrm{a}} \mathrm{C}$, crystal; $\mathrm{Sc}^{*}$, chiral smectic $\mathrm{C}$ phase; $\mathrm{S}_{\mathrm{A}}$, smectic A phase; $\mathrm{N}^{*}$ chiral nematic phase; I, isotropic phase

${ }^{\mathrm{b}}$ Response time to $10 \mathrm{~V} / \mu \mathrm{m}$ electric field at $25^{\circ} \mathrm{C}$ in a $2-\mu \mathrm{m}$ cell.

was observed only at temperatures below $46{ }^{\circ} \mathrm{C}$. The spontaneous polarization of the SCE8/CDH/TNF mixture is plotted as a function of temperature in Figure 18b. Similarly, the spontaneous polarization vanished when the temperature was raised above $46^{\circ} \mathrm{C}$. Thus, asymmetric energy exchange was observed only in the temperature range in which the sample exhibits ferroelectric properties, in other words, the $\mathrm{Sc}^{*}$ phase. Since the molecular dipole moment of FLCs is small and the dipole moment is aligned perpendicular to the molecular axis, large changes in the orientation of the molecular axis cannot be induced by the internal electric field in the $\mathrm{S}_{\mathrm{A}}$ or $\mathrm{N}^{*}$ phase of the FLCs. However, in the $\mathrm{Sc}^{*}$ phase, reorientation associated with spontaneous polarization occurs due to the internal electric field. The spontaneous polarization also causes the orientation of mesogens in the corresponding area to change accordingly. A maximum resolution of $0.8 \mu \mathrm{m}$ was obtained in this sample.

Several studies on the photorefractivity values of nematic LCs have been reported, wherein the resolution of refractive index grating was found to be low because of the multi-domain structure. Some of these studies indicate that only polymer-stabilized nematic LCs can form a Bragg diffraction grating. ${ }^{44,45}$ In many cases, nematic LCs are used in homeotropic alignment for photorefractivity studies. Although the homeotropic state is apparently transparent, the phase is composed of numerous small domains. Because of the fluid nature of nematic LCs, the directors of those domains fluctuate and cause dynamic light scattering. The formation of an internal electric field and orientational grating is affected by the multi-domain structure and results in low-resolution of the grating. Com- pared to nematic LCs, smectic phase LCs are more crystalline than liquid, therefore smectic LC films are highly transparent. A refractive index grating in the Bragg regime is readily formed in SS-FLCs.

\section{Comparison of Photorefractive Properties of FLCs}

The photorefractive properties of a series of FLCs were investigated, as shown in Table I. ${ }^{39}$ All the FLCs listed in Table I exhibited finely aligned SS-states when they were not mixed with $\mathrm{CDH}$ and TNF. Figure 19 shows typical examples of textures observed in the 017/000, M4851/050 and SCE8 FLCs. As the $\mathrm{CDH}$ concentration increased, defects appeared in the texture. M4851/050 and SCE8 retained the SSstate with few defects for $\mathrm{CDH}$ concentrations below $2 \mathrm{wt} \%$. All the FLCs listed in Table I, except for SCE8 and M4851/050, exhibited distorted SS-states, and light scattering was very strong when mixed with $\mathrm{CDH}$ at concentrations higher than $0.5 \mathrm{wt} \%$. SCE8 and M4851/050 displayed finely aligned SS-state domains in a $10 \mu \mathrm{m}$-gap cell and exhibited asymmetric energy exchange. FLCs that formed an SS-state with many defects did not exhibit clear asymmetric energy exchange. In these distorted SS-states, the laser beams are strongly scattered, precluding the formation of a refractive index grating.

The maximum gain coefficients of FLCs are plotted in Figure 20 as a function of the magnitude of spontaneous polarization. The formation of the refractive index grating is a process wherein the direction of spontaneous polarization of the areas between the light and dark positions of the interference fringes is altered. The formation of the refractive index grating must therefore be affected by the magnitude of spontaneous 

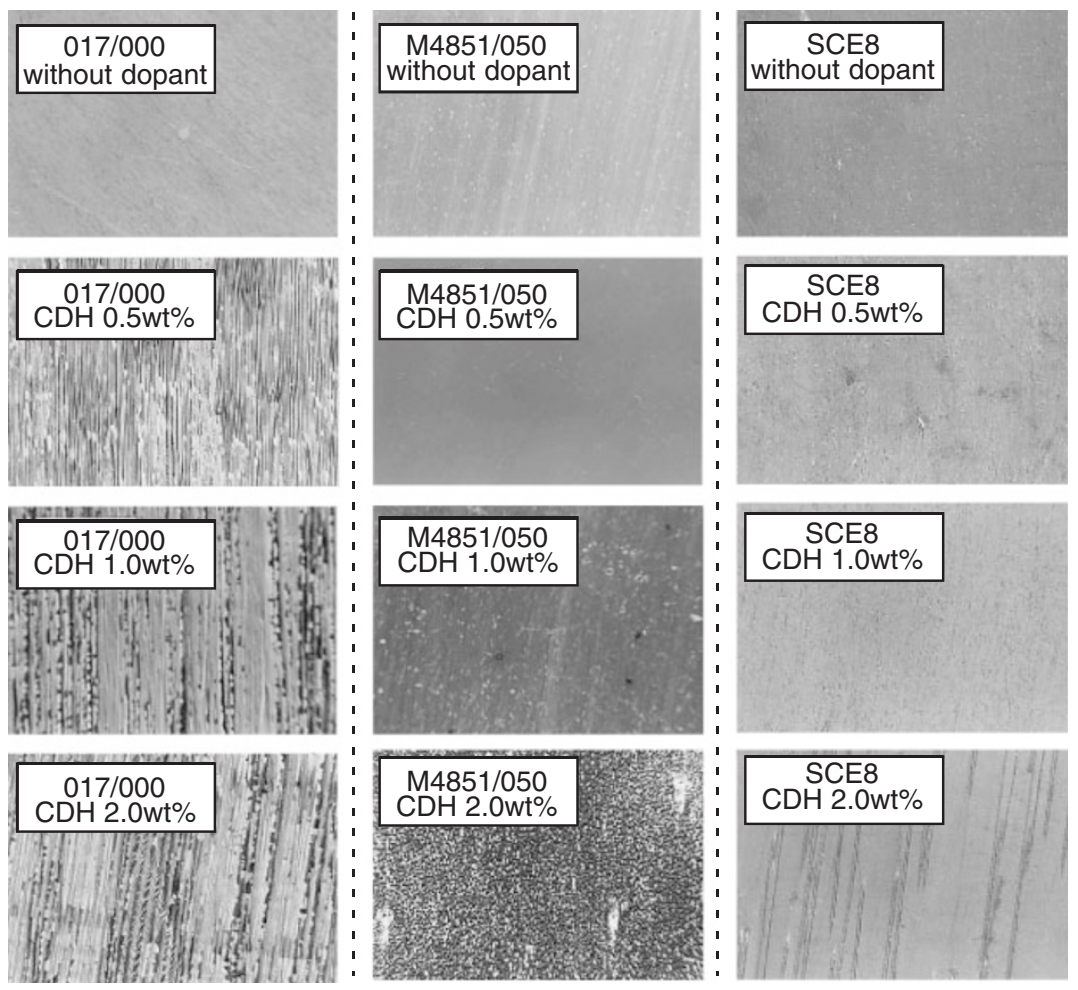

Figure 19. Textures observed by POM observation of SS-states in FLCs.

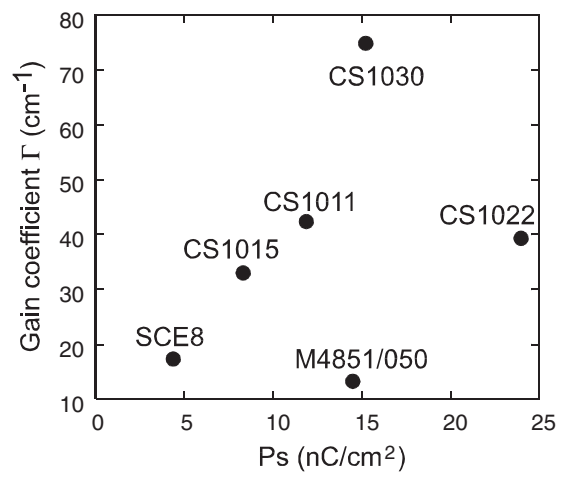

Figure 20. The maximum gain coefficients of FLCs under the application of a $0.1 \mathrm{~V} / \mu \mathrm{m}$ electric field as a function of the magnitude of spontaneous polarization.

polarization and the rotational viscosity. As seen in Figure 20, the gain coefficient is dependent on the magnitude of spontaneous polarization. Although the data points do not lie on a simple line, there is a tendency for the gain coefficient to be higher in FLCs with larger spontaneous polarization. This deviation may be caused by the differences in rotational viscosity, and the homogeneity of the SS-state in the samples.

\section{Effect of the Magnitude of Applied Electric Field}

The photorefractive effect in FLCs can be induced by applying a very weak external electric field. The maximum gain coefficient of the SCE8 sample was obtained using an electric field strength of only 0.2$0.4 \mathrm{~V} / \mu \mathrm{m}$. The dependence of the gain coefficient of
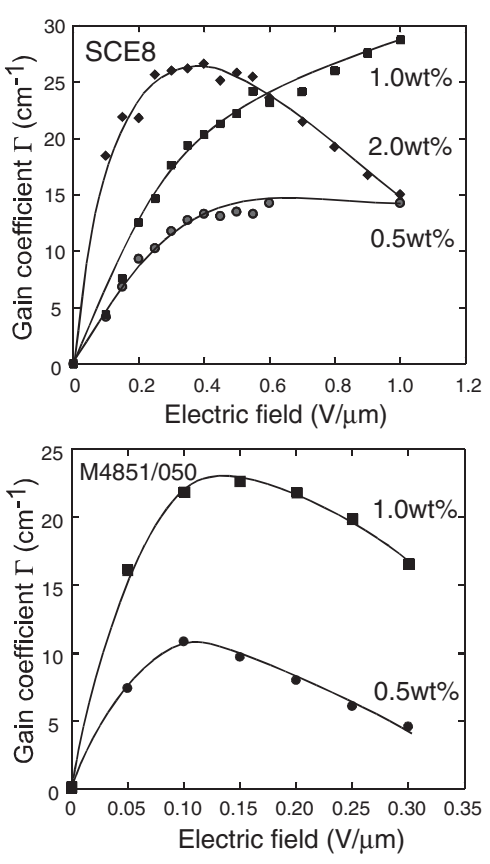

Figure 21. Electric field dependence of the gain coefficient of SCE8 and M4851/050 mixed with several concentrations of CDH and $0.1 \mathrm{wt} \% \mathrm{TNF}$ in a $10 \mu \mathrm{m}$-gap cell measured at $30^{\circ} \mathrm{C}$.

FLCs/CDH/TNF on the strength of the electric field is shown in Figure 21. The gain coefficient of SCE8 doped with $0.5-1 \mathrm{wt} \% \mathrm{CDH}$ increased with the strength of the external electric field. However, the gain coefficient of SCE8 doped with $2 \mathrm{wt} \% \mathrm{CDH}$ decreased when the external electric field exceeded 
$0.4 \mathrm{~V} / \mu \mathrm{m}$. The same tendency was observed for M4851/050 as well. The formation of the orientational grating is enhanced when the external electric field is increased from 0 to $0.2 \mathrm{~V} / \mu \mathrm{m}$ as a result of the induced charge separation under a higher external electric field. However, when the external electric field exceeded $0.2 \mathrm{~V} / \mu \mathrm{m}$, a number of zig-zag defects appeared in the texture. These defects cause light scattering and result in a decrease in the gain coefficient.

\section{Refractive Index Grating Formation Time}

The formation of the refractive index grating involves charge separation and reorientation. The index grating formation time is affected by these two processes, and both may be rate-determining steps. The refractive index grating formation times in SCE8 and M4851/050 were measured based on the simplest single-carrier model of photorefractivity, ${ }^{5}$ wherein the gain transient is exponential. The rising signal of the diffracted beam was fitted using a single exponential function as shown in eq 2.

$$
\gamma(t)-1=(\gamma-1)[1-\exp (-t / \tau)]
$$

Here, $\gamma(t)$ represents the transmitted beam intensity at time $t$ divided by the initial intensity $(\gamma(t)=I(t) / I 0)$ and $\tau$ is the formation time. The grating formation time in SCE8/CDH/TNF is plotted as a function of the strength of the external electric field in Figure 22a. The grating formation time decreased with increasing electric field strength due to the increased efficiency of charge generation. The formation time was shorter at higher temperatures, corresponding to a decrease in the viscosity of the FLC with increasing temperature. The formation time for SCE8 was found to be $20 \mathrm{~ms}$ at $30^{\circ} \mathrm{C}$. As shown in Figure $22 \mathrm{~b}$, the formation time for M4851/050 was found to be independent of the magnitude of the external electric field, with a formation time of $80-90 \mathrm{~ms}$ for M4851/050 doped with $1 \mathrm{wt} \% \mathrm{CDH}$ and $0.1 \mathrm{wt} \% \mathrm{TNF}$. This is slower than for SCE8, although the spontaneous polarization of $\mathrm{M} 4851 / 050\left(-14 \mathrm{nC} / \mathrm{cm}^{2}\right)$ is larger than that of SCE8 $\left(-4.5 \mathrm{nC} / \mathrm{cm}^{2}\right)$, and the response time of the electro-optical switching (the flipping of spontaneous polarization) to an electric field $( \pm 10 \mathrm{~V}$ in a $2 \mu \mathrm{m}$ cell $)$ is shorter for M4851/050. The slower formation of the refractive index grating must be due to the poor homogeneity of the SS-state in M4851/050 and the charge mobility.

\section{Effect of the Beam Intensity}

The dependence of the gain coefficients of SCE8/ $\mathrm{CDH} / \mathrm{TNF}$ on the beam intensity is shown in Figure 23. The gain coefficient increased with the beam intensity up to $255 \mathrm{~mW} / \mathrm{cm}^{2}$, and then become almost constant at higher intensities. This can be explained
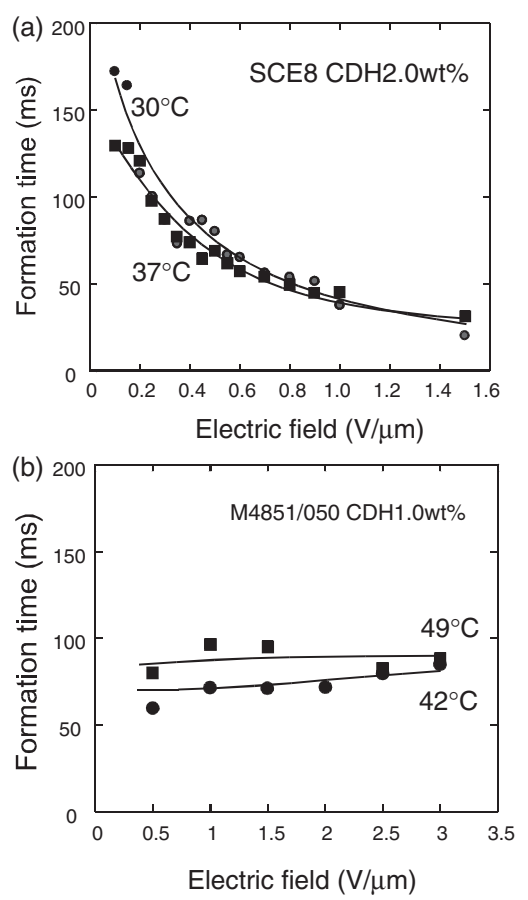

Figure 22. Electric field dependence of index grating formation time. (a) SCE8 mixed with $2 \mathrm{wt} \% \mathrm{CDH}$ and $0.1 \mathrm{wt} \%$ TNF in two-beam coupling experiment. $\bullet$, measured at $30^{\circ} \mathrm{C}$ $\left(T / T_{\mathrm{Sc}^{*}-\mathrm{S}_{\mathrm{A}}}=0.95\right) ; \boldsymbol{\square}$, measured at $36^{\circ} \mathrm{C}\left(T / T_{\mathrm{Sc}^{*}-\mathrm{S}_{\mathrm{A}}}=0.97\right)$. (b) M4851/050 mixed with $1 \mathrm{wt} \% \mathrm{CDH}$ and $0.1 \mathrm{wt} \% \mathrm{TNF}$ in a two-beam coupling experiment. - measured at $42^{\circ} \mathrm{C}$ $\left(T / T_{\mathrm{Sc}^{*}-\mathrm{S}_{\mathrm{A}}}=0.95\right) ; \boldsymbol{\square}$, measured at $49{ }^{\circ} \mathrm{C}\left(T / T_{\mathrm{Sc}^{*}-\mathrm{S}_{\mathrm{A}}}=0.97\right)$.

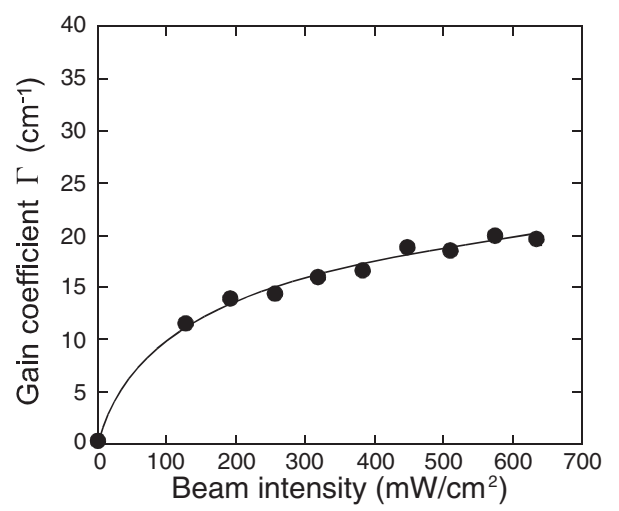

Figure 23. Dependence of the gain coefficient of the FLC/ $\mathrm{CDH} / \mathrm{TNF}$ mixture on the beam intensity.

by the refractive index grating, which is caused by the generation of a space-charge field. When the intensity of the beam is low, the strength of the spacecharge field increases as the intensity increases, because of the increased amount of generated charge. The strength of the space-charge field then becomes saturated at sufficiently high beam intensities.

The effect of the beam intensity on the refractive index grating formation time was also investigated. The formation time is plotted as a function of the beam intensity in Figure 24. The formation time was 


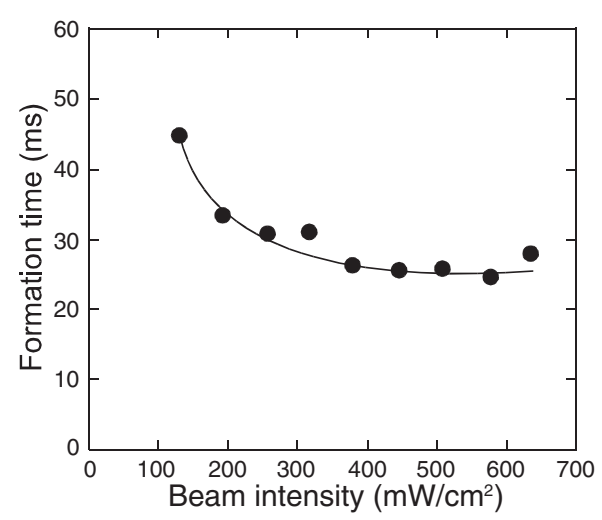

Figure 24. The index grating formation time of the FLC/ $\mathrm{CDH} / \mathrm{TNF}$ mixture as a function of the beam intensity.

shortened as the intensity of the beam increased because of the accelerated formation of the space charge field, and then became constant at intensities higher than $380 \mathrm{~mW} / \mathrm{cm}^{2}$.

\section{Formation of Dynamic Holograms Based on Spatial Modulation of Molecular Motions of FLCs}

The formation of dynamic holograms based on the spatial modulation of the molecular motion of ferroelectric liquid crystals (FLCs) was demonstrated. ${ }^{42}$ The switching movement of an FLC molecule is essentially a rotational motion along a conic surface. ${ }^{59}$ When an alternating triangular-waveform voltage is applied, the FLC molecules uniformly perform a consecutive rotational switching motion along a conic surface (Figure 25a). If a photoconductive FLC material is used, the rotational motion can be modulated by illuminating it with interfering laser beams, as shown in Figure 25. The internal electric field vector is directed along the interference fringe wave vector and, in many cases, it differs from the direction of the applied alternating electric field. Thus, the total electric field at each moment on the FLC molecules is altered by the presence of the internal electric field. The consecutive rotational motion of the FLC molecules in the areas between the light and the dark positions of the interference fringes is biased by the internal electric field. Consequently, a grating based on the spatial difference in the rotational motion (or switching motion) of the FLC molecules is created. This grating is different from those used currently for holograms wherein changes in the static properties of a medium, such as absorbance, transparency, film thickness, molecular orientation etc., are induced by photochemical reactions.

The formation of a motion-mode grating was examined using a SCE8/CDH/TNF mixture in a $10 \mu \mathrm{m}$ gap-cell. ${ }^{42}$ The formation of a holographic grating and the existence of a phase shift between the formed (a)

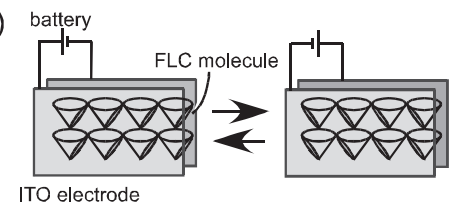

(b)

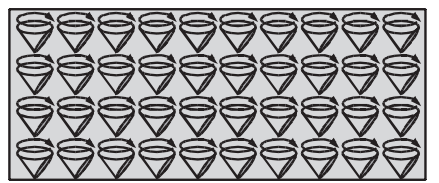

(c) dark

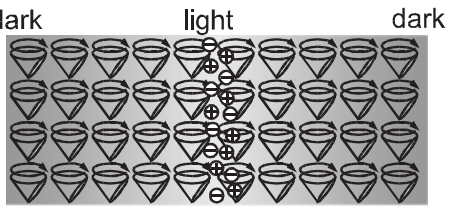

(d) dark

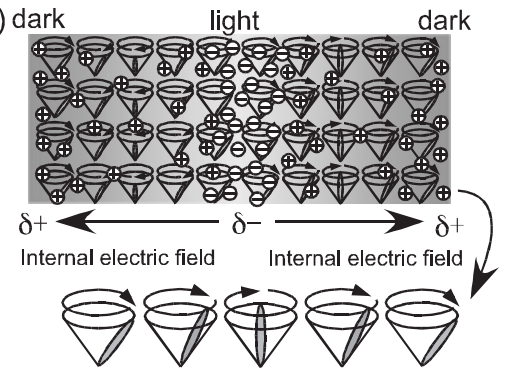

Figure 25. (a) Electro-optical switching of an FLC. (b) The rotational motion of FLC molecules under the application of an alternating electric field. (c) Positive and negative charges appear at the light positions of the interference fringe. (d) An internal electric field develops in the area between the light and dark positions of the interference fringes. The rotational motion of FLC molecules in the corresponding area is biased by the internal electric field.

grating and the interference fringes were examined in this experiment. An alternating triangular-waveform electric field $(0- \pm 1 \mathrm{~V} / \mu \mathrm{m}$, and $1 \mathrm{kHz}-3 \mathrm{MHz})$ was applied to the sample. Under the effect of an alternating triangular-waveform electric field of \pm 0.5 $\mathrm{V} / \mu \mathrm{m}, 100 \mathrm{kHz}$ in the two-beam coupling experiment, the FLC molecules showed consecutive switching motion.

Figure 26 shows the transmitted intensities of two laser beams passing through the sample under the effect of an alternating electric field as a function of time. Interference of the divided beams in the sample resulted in increased transmittance of one beam and decreased transmittance of the other. The change in transmitted intensities of the two beams is completely symmetric, as can be seen in Figure 26. This indicates that a grating based on the spatial difference in the rotational switching motion of FLC molecules was formed and acted as a diffraction grating. The spacing of the grating was calculated to be $0.9 \mu \mathrm{m}$ (1100 lines/ $\mathrm{mm}$ ). The gain coefficient was almost independent of the external electric field strength. This behavior is different from the photorefractive effect in FLCs 


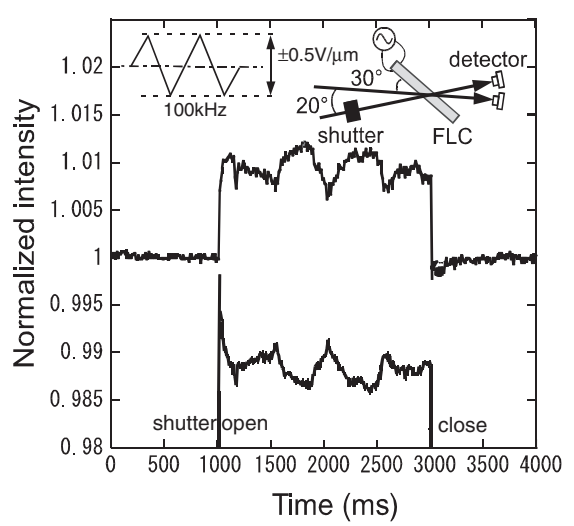

Figure 26. Typical example of the asymmetric energy exchange observed in two-beam coupling experiments. A triangular waveform electric field with $\pm 0.5 \mathrm{~V} / \mu \mathrm{m}$ and $100 \mathrm{kHz}$ was applied in this case.

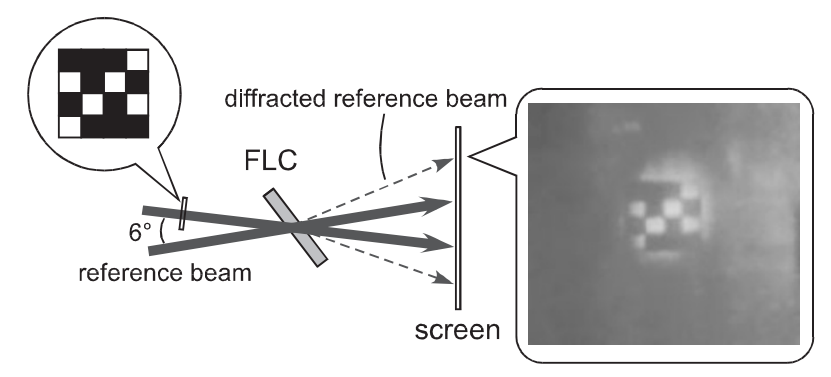

Figure 27. Photograph of an optical image diffracted by a motion-mode hologram. An electric field of $\pm 0.5 \mathrm{~V} / \mu \mathrm{m}$ and 100 $\mathrm{kHz}$ was applied.

under an applied DC electric field (Figure 21). The grating formation time was found to be $18-32 \mathrm{~ms}$.

The writing of optical images using the motionmode hologram was experimentally investigated. The optical image and the reference beam were made to interfere in the sample in the Raman-Nath (thin hologram) condition. ${ }^{4}$ In this condition, higher order diffractions are allowed. A laser beam transmitted through a transparent sheet printed with a pattern (object beam) was interfered with a reference beam in the FLC. A part of the reference beam was diffracted by the hologram into the angle of higher order diffractions. Figure 27 shows the photograph of the first order diffraction on a screen. Clearly, the diffracted reference beam contains information about the image, indicating that a motion-mode hologram image was formed in the FLC medium. The first-order diffraction efficiency was measured to be $0.19 \%$. The motionmode grating vanishes when the internal electric field disappears. The separated charges recombine quickly when the interference fringes disappear. Thus, the motion-mode grating is reversible and can be utilized for dynamic real-time holograms such as phase conjugate mirrors and optical signal amplifiers.

\section{CONCLUSIONS}

The photorefractive properties of a series of sidechain polymers and a series of FLC mixtures were investigated. The diffraction efficiencies of the isotropic phases of liquid crystalline polymers were compared with those of amorphous polymers. The diffraction efficiency was found to be much larger in the isotropic phase of the liquid crystalline polymers compared to those in amorphous polymers. The enhancement of photorefractivity is considered to be caused by the aligned structure-forming nature of the isotropic phase of liquid crystalline polymers and high transparency. The LC polymer that included a hydrogen-bonding moiety exhibited large diffraction efficiencies at temperatures below the glass transition temperature. The origin of this larger diffraction efficiency at lower temperatures was considered to be a larger free-volume distribution and the existence of micro-domain structures.

A re-orientational photorefractive effect based on the response of bulk polarization was observed in dye-doped FLC samples. Photorefractivity was observed only in the ferroelectric phase of these samples, and the refractive index formation time was found to be shorter than that for nematic LCs. The response time was in the order of a few tens of ms and is dominated by the formation of the internal electric field. These results indicate that the mechanism responsible for refractive index grating formation in FLCs is different from that for non-ferroelectric materials, and is clearly related to the ferroelectric properties of the material. The photorefractive properties of FLCs was strongly affected by the properties of the FLCs. Besides properties such as spontaneous polarization, viscosity, and phase transition temperature, the homogeneity of the SS-state was also found to be a major factor. The gain coefficient, refractive index grating formation time (response time) and stability of the two-beam coupling signal were all affected strongly by the homogeneity of the SS-state. Therefore, a highly homogeneous SS-state is necessary to create a photorefractive device. The techniques employed recently in the development of FLC display panels and defect-free SS-FLC panels, ${ }^{34,35}$ will be utilized in the future for the fabrication of photorefractive devices.

Acknowledgment. The author thanks Prof. M. Ueda, Editor-in-Chief, for inviting us to write this review article. This work has been partially supported by a Grant-in-Aid for Scientific Research from the Ministry of Education, Culture, Sports, Science and Technology. 


\section{REFERENCES}

1. F. S. Chen, J. Appl. Phys., 38, 3418 (1967).

2. K. Shutter, J. Hulliger, and P. Gunter, Solid State Commun., 74, 867 (1990).

3. L. Solymar, J. D. Webb, and A. Grunnet-Jepsen, "The Physics and Applications of Photorefractive Materials," Oxford, New York, 1996.

4. P. Yeh, "Introduction to Photorefractive Nonlinear Optics," John Wiley, New York, 1993.

5. W. E. Moerner and S. M. Silence, Chem. Rev., 94, 127 (1994).

6. B. Kippelen and N. Peyghambarian, "Advances in Polymer Science, Polymers for Photonics Applications II: Nonlinear Optical, Photorefractive and Two-Photon Absorption Polymers," Springer, 2002.

7. O. Ostroverkhova and W. E. Moerner, Chem. Rev., 104, 3267 (2004).

8. Y. Zhang, Y. Cui, and P. N. Preasad, Phys. Rev. B, 46, 9900 (1992).

9. B. Kippelen, Sandalphon, N. Peyghambarian, S. R. Lyon, A. B. Padias, and H. K. Hall Jr., Electron Lett., 29, 1873 (1993).

10. W.-K. Chan, Y. Chen, Z. Peng, and L. Yu, J. Am. Chem. Soc., 115, 11735 (1993).

11. Z. Peng, Z. Bao, and L. Yu, J. Am. Chem. Soc., 116, 6003 (1994).

12. K. Meerholz, B. L. Volodin, B. Kippelen, and N. Peyghambarian, Nature, 371, 497 (1994).

13. B. L. Volodin, B. Kippelen, K. Meerholz, B. Javidi, and N. Peyghambarian, Nature, 383, 58 (1996).

14. Z. Peng, A. R. Gharavi, and L. Yu, J. Am. Chem. Soc., 119, 4622 (1997).

15. Y. Cui, B. Swedek, N. Cheng, K. S. Kim, and P. N. Prasad, J. Phys. Chem. B, 101, 3530 (1997).

16. A. Grunnet-Jepsen, C. L. Thompson, and W. E. Moerner, Science, 277, 549 (1997).

17. B. Kippelen, S. R. Marder, E. Hendrickx, J. L. Maldonado, G. Guillemet, B. L. Volodin, D. D. Steele, Y. Enami, Sandalphon, Y. J. Yao, J. F. Wang, H. Röckel, L. Erskine, and N. Peyghambarian, Science, 279, 54 (1998).

18. K. Meerholz, Y. D. Nardin, R. Bittner, R. Wortmann, and F. Würthner, Appl. Phys. Lett., 73, 4 (1998).

19. E. Hendrickx, J. F. Wang, J. L. Maldonado, B. L. Volodin, E. A. Mash, A. Persoons, B. Kippelen, and N. Peyghambarian, Macromolecules, 31, 734 (1998).

20. E. Hattemer, R. Zentel, E. Mecher, and K. Meerholz, Macromolecules, 33, 1972 (2000).

21. D. V. Steenwinckel, C. Engels, E. Gubbelmans, E. Hendrikx, C. Samyn, and A. Persoons, Macromolecules, 33, 4074 (2000).

22. L. Yu, J. Polym. Sci.; Part A: Polym. Chem., 39, 2557 (2001).

23. D. Wright, U. Gubler, W. E. Moerner, M. S. CeClue, and J. S. Siegel, J. Phys. Chem. B, 107, 4732 (2003).

24. L. C. Khoo, H. Li, and Y. Liang, Opt. Lett., 19, 1723 (1994).

25. I. C. Khoo, "Liquid Crystals: Physical Properties and Nonlinear Optical Phenomena,” Wiley, New York, N.Y., 1995.
26. G. P. Wiederrecht, B. A. Yoon, and M. R. Wasielewski, Science, 270, 1794 (1995).

27. G. P. Wiederrecht, B. Yoon, W. A. Svec, and M. R. Wasielewski, J. Am. Chem. Soc., 119, 3358 (1997).

28. G. P. Wiederrecht and M. R. Waiselewski, J. Am. Chem. Soc., 120, 3231 (1998).

29. T. Sasaki, S. Hamada, Y. Ishikawa, and T. Yoshimi, Chem. Lett., 1183 (1997).

30. T. Sasaki, M. Goto, Y. Ishikawa, and T. Yoshimi, J. Phys. Chem. B, 103, 1925 (1999).

31. T. Sasaki, Proc. SPIE, 3799, 14 (1999).

32. T. Sasaki, K. Tachibana, K. Ohno, T. Shimada, M. Kudo, A. Katsuragi, and T. Furuta, Mol. Cryst. Liq. Cryst., 368, 345 (2001).

33. T. Sasaki, T. Shimada, and K. Tachibana, Chem. Lett., 31, 324 (2002).

34. T. Sasaki, R. Kai, A. Sato, Y. Ishikawa, and T. Yoshimi, Mol. Cryat. Liq. Cryst., 373, 53 (2002).

35. T. Sasaki and G. Fukunaga, Chem. Mater, 2005, in press.

36. T. Sasaki, Y. Kino, M. Shibata, N. Mizusaki, A. Katsuragi, Y. Ishikawa, and T. Yoshimi, Appl. Phys. Lett., 78, 4112 (2001).

37. T. Sasaki, A. Katsuragi, and K. Ohno, J. Phys. Chem. B, 106, 2520 (2002).

38. T. Sasaki, K. Ohno, and Y. Nakazawa, Macromolecules, 35, 4317 (2002).

39. T. Sasaki, A. Katsuragi, O. Mochizuki, and Y. Nakazawa, J. Phys. Chem. B, 107, 7659 (2003).

40. T. Sasaki, O. Mochizuki, K. Noborio, and Y. Nakazawa, J. Phys. Chem. B, 108, 17083 (2004).

41. Y. Nakazawa and T. Sasaki, Chem. Lett., 33, 242 (2004).

42. T. Sasaki, O. Mochizuki, Y. Nakazawa, G. Fukunaga, T. Nakamura, and K. Noborio, Appl. Phys. Lett., 85, 1329 (2004)

43. H. Ono, T. Kawamura, N. M. Frias, K. Kitamura, N. Kawatsuki, and H. Norisada, Adv. Mater., 12, 143 (2000).

44. H. Ono and N. Kawatsuki, Opt. Lett., 22, 1144 (1997).

45. A. Golemme, B. L. Bolodin, B. Kippelen, and N. Peyghambarian, Opt. Lett., 22, 1226 (1997).

46. H. Ono and N. Kawatsuki, Jpn. J. Appl. Phys., 36, 6444 (1997).

47. H. Ono, I. Sato, and N. Kawatsuki, Appl. Phys. Lett., 72, 1942 (1998).

48. H. Ono and N. Kawatsuki, Jpn. J. Appl. Phys., 38, 737 (1999).

49. H. Ono, A. Hanazawa, T. Kawamura, H. Norisada, and N. Kawatsuki, J. Appl. Phys., 86, 1785 (1999).

50. H. Ono and N. Kawatsuki, J. Appl. Phys., 85, 2482 (1999).

51. H. Ono, T. Kawamura, N. Kawatsuki, H. Norisada, and T. Yamamoto, Jpn. J. Appl. Phys., 38, L1258 (1999).

52. H. Ono and N. Kawatsuki, J. Nonlinear, Opt. Phys. Mater., 8, 329 (1999).

53. H. Ono, T. Kawamura, N. M. Frias, K. Kitamura, N. Kawatsuki, H. Norisada, and T. Yamamoto, J. Appl. Phys., 88, 3853 (2000).

54. H. Ono, T. Kawamura, N. M. Frias, K. Kitamura, N. Kawatsuki, and H. Norisada, Adv. Mater., 12, 143 (2000).

55. H. Ono, K. Kitamura, N. Kawatsuki, and H. Norisada, Jpn. J. Appl. Phys., 40, 1328 (2001). 
56. U. Hofmann, M. Grasruck, A. Leopold, A. Schreiber, S. Schloter, C. Hohle, P. Strohriegl, D. Haarer, and S. J. Zilker, J. Phys. Chem. B, 104, 3887 (2000).

57. O. Ostroverkhova and W. E. Moerner, Appl. Phys. Lett., 82, 3602 (2003).

58. K. Skarp and M. A. Handschy, Mol. Cryst. Liq. Cryst., 165,
439 (1988).

59. A. Fukuda and H. Takezoe, "Structure and Properties of Ferroelectric Liquid Crystals," Corona, Tokyo, 1990.

60. Y. Ouchi, H. Takezoe, and A. Fukuda, Jpn. J. Appl. Phys., 26, 4 (1987).

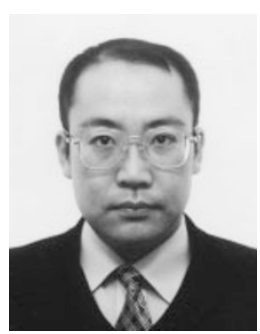

Takeo Sasaki was born in Hamamatsu in 1965. He received Ph.D. from Tokyo Institute of Technology in 1994 for a study on the photochemical control of properties of liquid crystals. He joined Tohoku University as a Research Associate at Professor Minoru Matsuda's laboratory, where he worked on nonlinear optical properties of polymers. In 1996, he joined Oita University as an Associate Professor and started the studies on photorefractive materials. Since 2000, he has been in Science University of Tokyo as an Associate Professor. He received SPSJ Wiley Award (2004) from the Society of Polymer Science, Japan. His current research interests include photorefractive effect of organic materials and synthesis of novel liquid crystalline materials. 